Results Among the 979 charts reviewed, only 278 patients were included in the analysis. Seventy-five percent of the patients had squamous cell carcinoma and only $20 \%$ had adenocarcinoma. Baseline characteristics were comparable. Ninety-eight percent had Cisplatin-based concurrent chemoradiotherapy. Median follow up was 17 months, with $75.30 \%$ of the patients had complete response, 7.97 had partial response and $16.73 \%$ had recurrent disease. Patients having squamous cell carcinoma had higher percentage of being alive at the time of follow up, better response to treatment, lesser recurrence and lower mortality rate as compared to adenocarcinoma, however, there was no sufficient evidence to demonstrate a difference in disease free survival and overall survival. Conclusion Patients with locally advanced adenocarcinoma of the cervix who underwent concurrent chemoradiation had the same treatment response and prognosis to patients with squamous cell carcinoma.

Disclosures None.

\section{PROGNOSTIC IMPACT OF SERUM INFLAMMATORY BIOMARKERS COMBINED WITH IL-6 EXPRESSION IN CERVICAL CANCER PATIENTS}

'Lavinia Domenici, ${ }^{2}$ Silvia Garibaldi, ${ }^{2}$ Alessandra Perutelli, ${ }^{3}$ Alessandro Tonacci, ${ }^{2}$ Pietro Bottone, 'Ludovico Muzii, 'Pierluigi Benedetti Panici. 'University "Sapienza" of Rome; Division of Obstetrics and Gynecology, Department of Clinical and Experimental Medicine, University of Pisa, Pisa; Department of Gynecological, Obstetrical and Urological Sciences; ${ }^{2}$ University of Pisa; Division of Obstetrics and Gynecology, Department of Experimental and Clinical Medicine; ${ }^{3}$ National Research Council; Institute of Clinical Physiology (Cnr-Ifc)

\subsection{6/ijgc-2020-ESG0.203}

Introduction/Background Increasing evidences demonstrated a crucial role of inflammation in inducing and promoting several cancers. Cancer-related inflammation is an essential process in malignant disease by stimulating tumour cells proliferation, invasion mechanisms, metastasis, neoangiogenesis and by activating pathways of apoptosis's resistance. Cells and mediators of inflammation (as cytokines) represent a major part of tumour milieu. Particularly, IL-6 has been linked with cervical cancer development and progression by inducing upregulation of vascular growth factors (as VEGF), by modulating apoptosis, by fostering platelet production, activation and aggregation. An elevated platelet to lymphocyte ratio (PLR) has been recognised as markers of inflammation and also linked to poor prognosis in several malignancies. The aim of the study

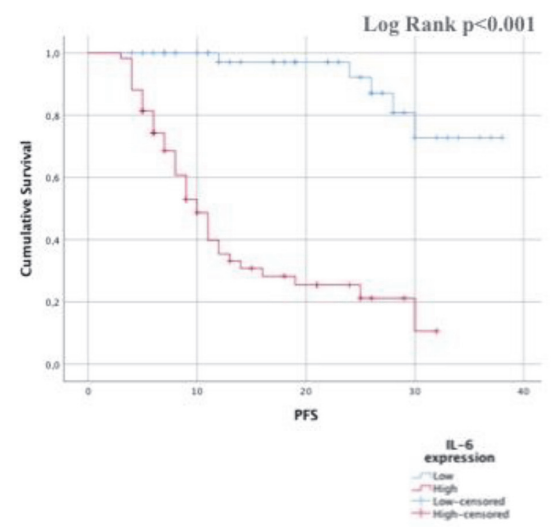

was to evaluate prognostic impact of inflammatory biomarkers (high platelet count, PLR) in combination with IL-6 tumour expression in cervical cancer patients.

Methodology Between 2016 and 2019, 108 out of 159 patients with cervical cancer presented to the Department of Gynecological, Obstetrical and Urological Sciences of "Sapienza" University of Rome and to the Division of Obstetrics and Gynecology at Department of Experimental and Clinical Medicine, University of Pisa have been enrolled. Study project was made in collaboration with National Research Council of Italy, Institute of Clinical Physiology (CNR-IFC) of Pisa. Cut off level of pre-treatment platelet count and PLR were identified by using ROC curve. IL- 6 tumoural and peri-tumoural expression was analysed and stratified as low and high (low expression: $0,+1$; marked expression: $+2,+3)$.

Results Median follow up duration was 30 months (range 1644). Patients with higher platelet counts showed worse OS and DFS (OS p $<0.001$ and DFS $\mathrm{p}<0.001$, respectively figure $1 \mathrm{~A})$. Cumulative rates of OS and DFS in patients with lower PLR were higher than in patients with higher values of PLR (OS $\mathrm{p}<0.001$ and DFS $\mathrm{p}=0.032$; figure 1B). Survival analysis showed a better prognosis in patients with lower IL-6 tissutal expression (PFS $\mathrm{p}<0.001$; OS $\mathrm{p}<0.001$; figure 2).

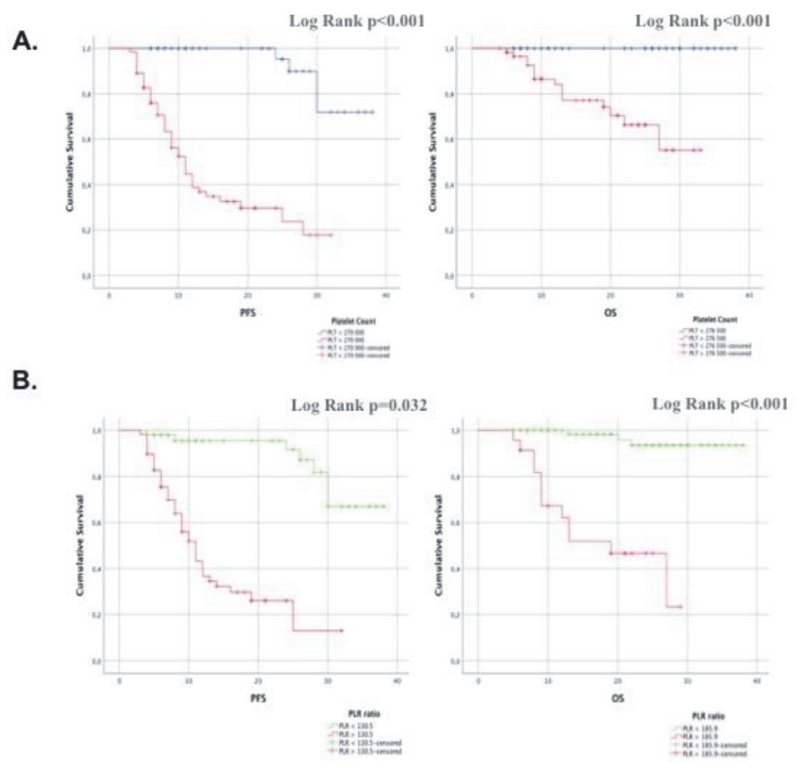

Abstract 583 Figure 1

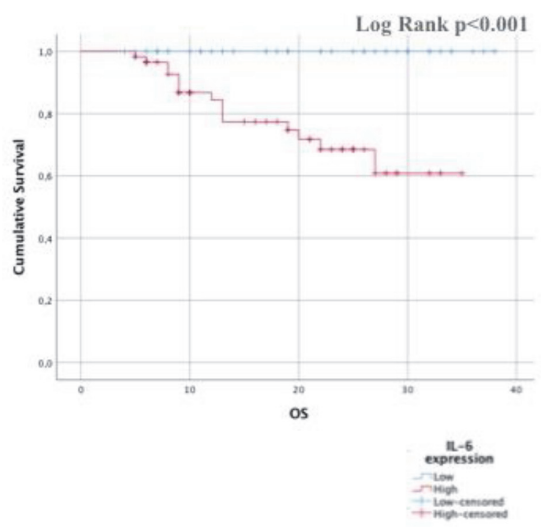


Patients' characteristics were stratified according to platelet count, PLR, and IL-6 tissutal expression then compared using Pearson's correlation. Significant correlations were observed between negative cervical cancer-related prognostic factors (advanced stage of disease, tumor size, high grading, positive LVSI, lymph nodes and parametrial involvement) and proinflammatory patient's status.

Conclusion Nowadays causal relationship between inflammation, innate immunity and cancer is more widely accepted; however, many of the molecular and cellular mechanisms mediating this relationship remain unresolved.

Ongoing inflammatory response was associated with poor outcomes in cervical cancer patients. A higher pre-treatment platelet count and PLR value associated with higher IL-6 tumoral expression could be used to predict poor prognosis in cervical cancer patients. Further investigations about inflammatory markers in prognostic models could contribute in early cervical patients' stratification and consequent management.

Disclosures None.

\section{SENTINEL LYMPH NODE MAPPING IN EARLY-STAGE CERVICAL CANCER USING NEAR-INFRARED FLUORESCENCE IMAGING: A PROSPECTIVE PILOT STUDY}

${ }^{1}$ Anastasios Tranoulis, ${ }^{2}$ Hebatallah Awad, ${ }^{2}$ Christina Thompson, ${ }^{2}$ Amy Fisher, ${ }^{2}$ Jeremy Twigg. ${ }^{1}$ The Pan-Birmingham Gynaecological Oncology Centre, Sandwell and West Birmingham NHS Trust, Birmingham; ${ }^{2}$ Jame's Cook University Hospital, Middlesbrough

\subsection{6/ijgc-2020-ESGO.204}

Introduction/Background The aim of this pilot study was to ascertain the feasibility of sentinel lymph node (SLN) mapping in early-stage cervical cancer (CC) and evaluate factors affecting bilateral SLN detection.

Methodology This was a prospective cohort study spanning the period from January 2015 to March 2019. Women with early-stage CC (FIGO 2018 stage IA2 to IB2) scheduled to undergo robot-assisted laparoscopic radical hysterectomy or trachelectomy and SLN mapping with the Da Vinci Si Surgical System with near-infrared (NIR) imaging and indocyanine (ICG) fluorescence detection were enrolled. All patients were given the choice of complete pelvic lymph node dissection and SLN mapping or SLN mapping only. Cervical injection with ICG $(2 \mathrm{ml})$ was performed for all women. Side-specific lymphadenectomy was performed when mapping was unsuccessful. The detection rate was calculated. Plausible correlations amongst the variables were examined using the Spearman's correlation coefficient (rho) and multivariate logistic regression.

Results The study enrolled 47 women [mean age 41.83 (24-69) years; mean body mass index (BMI) 29.02 (19-48) $\mathrm{Kg} / \mathrm{m} 2$ ], of whom 43 underwent robot-assisted laparoscopic radical hysterectomy, whilst four radical trachelectomy. 31 $(65.95 \%)$ women were diagnosed with squamous cell carcinoma, while $13(27.69 \%)$ with adenocarcinoma, 2 (4.25\%) with adeno-squamous cell carcinoma and 1 (2.11\%) with endometrioid carcinoma, respectively. In total, 76 SLNs were removed. SLNs were most commonly identified in the external iliac basins (68.4\%), followed by the obturator fossa $(21.05 \%)$, internal iliac basins (6.6\%) and common iliac basins (3.95\%), respectively. SLN detection rates were
$87.23 \%$ per patient, $84.05 \%$ per heme-pelvis and $68.09 \%$ bilaterally. Lymph node metastasis was detected in 3 women (6.3\%). There was no statistical correlation between bilateral SLN detection and age (rho=-0.09, $\mathrm{p}=0.63)$, BMI (rho $=-0.08,0.57)$, adenocarcinoma or adeno-squamous histological sub-type $(\mathrm{rho}=0.14, \mathrm{p}=0.35)$, grade 3 (rho=-0.09, $\mathrm{p}=0.55)$ or tumour size $>2 \mathrm{~cm}$ $(\mathrm{rho}=0.19, \mathrm{p}=0.21)$. None of these covariates were significant in multivariate analysis.

Conclusion Intra-operative SLN mapping using fluorescence imaging with ICG is feasible, yields high diagnostic accuracy and it can be considered as an alternative to complete lympadenectomy for carefully selected patients with early-stage CC.

Disclosures We certify that no party has a direct interest in the results of the research and that no benefit will be conferred to us or any organisation with which we are associated.

\section{ASSESSING TRENDS IN STAGE AND OUTCOMES OF UTERINE CERVIX CANCER IN AN OPPORTUNISTIC SCREENING SETTING}

${ }^{1}$ Priscila Tavares Cruz Lugarinho, 'Silvana Maria Quintana, 'Jurandyr Moreira de Andrade, ${ }^{1}$ Daniel Guimarães Tiezzi, ${ }^{2}$ Francisco Jose Candido Dos Reis. ' Ribeirão Preto Medical School/ University of São Paulo; ${ }^{2}$ Ribeirao Preto Medical School; Gynecology and Obstetrics

\subsection{6/ijgc-2020-ESG0.205}

Introduction/Background Organized screening programs reduce cervical cancer incidence and mortality. However, in many low-middle income countries, the screening programs are opportunistic. In this work, we investigated the trends in cervical cancer mortality, advanced-stage at diagnosis, and screening coverage in an opportunistic setting.

Methodology In this large retrospective cohort, we analyzed data on invasive cervical cancer diagnosed between January 2000 and December 2014. Cancer data were provided by Fundação Oncocentro de São Paulo (FOSP) and screening coverage by the Instituto Brasileiro de Geografia e Estatistica (IBGE). Five-year cervical mortality was calculated using the Fine and Gray regression model. Joinpoint regression analysis was used to estimate annual percentage changes (APC) for five-year mortality, the proportion of advanced stage, and screening coverage.

Results From 18,206 cases, we identified 6,479 deaths in five years of follow up due to cervical cancer. The leading risk factor was the advanced stage $(\mathrm{sHR}=6.48,95 \%$ CI 5.75 to 7.30). The rate of cervical cancer mortality was stable from 2000 to 2014 (APC $=-0.106 ; 95 \% \mathrm{CI}-0.730$ to 0.522 ; $\mathrm{P}=0.720$ ). The estimated proportion of women screened (in the last three years) was $81 \%$ in $2003,83 \%$ in 2008 , and $85 \%$ in $2013 \quad(\mathrm{APC}=0.443 ; 95 \% \mathrm{CI}-4.913$ to 6.102 ; $\mathrm{P}=0.492)$. The proportion of advanced stage reduced from $77 \%$ in 2000 to $71 \%$ in 2006 (APC $=-1.294 ; 95 \%$ CI -2.322 to $-0.256 ; \mathrm{P}=0.020)$ and increased to $76 \%$ in 2014 ( $\mathrm{APC}=0.924 ; 95 \% \mathrm{CI} 0.248$ to $1.605 ; \mathrm{P}=0.012$ ).

Conclusion Opportunistic screening strategies fail to achieve a similar proportion of early-stage diagnosis and reduced mortality in invasive uterine cervix cancer compared to organized screening programs.

Disclosures The authors have no conflict of interest to disclose. 